\title{
HIV-associated sensory neuropathy in HIV-1 infected patients at the Douala General Hospital in Cameroon: a cross-sectional study
}

Henry Namme Luma ${ }^{1,3^{*}}$, Benjamin Clet Nguenkam Tchaleu ${ }^{1,4}$, Marie Solange Doualla ${ }^{1,3}$, Elvis Temfack', Victor Nicolas King Sopouassi ${ }^{4}$, Yacouba Njankouo Mapoure ${ }^{1}$ and Vincent-de-Paul Djientcheu ${ }^{2,3}$

\begin{abstract}
Background: Peripheral neuropathy (PN) which is the most common neurological complication of HIV infection is under recognised and undertreated especially in resource limited settings. This ailment which has a negative impact on the quality of life of HIV/AIDS patients exists in different clinical patterns of which HIV-associated Sensory neuropathy (HIV-SN) is the most common affecting up to two thirds of patients with advanced disease in some settings. In Cameroon where HIV is a major public health problem, the burden of HIV-SN has not yet been well defined.

Methods: Using the Brief Peripheral Neuropathy Screening (BPNS) tool validated by the AIDS Clinical Trial Group (ACTG) we carried out a cross sectional study to determine the prevalence of HIV-SN and its associated factors among HIV-1 patients at the Douala General Hospital between $1^{\text {st }}$ July and $31^{\text {st }}$ October 2011. HIV-SN was defined as the presence of neuropathic symptoms and at least an abnormal perception of vibrations of a $128 \mathrm{~Hz}$ tuning fork on the great toe or abnormal ankle reflexes or both and expressed as a percentage of the study population.

Results: Out of 295 patients studied, $21 \%$ had HIV-SN. In HIV-SN patients the median duration of HIV infection was 79.8 months (IQR 46 - 107.5) and their median CD4 count 153cells/ML (IQR 80 - 280). Patient recall and clinical chart review showed that, $83.9 \%$ had neuropathic symptoms prior to HAART initiation and $16.1 \%$ after HAART initiation. Low CD4 count, history of alcohol intake and history of anti-tuberculosis treatment were strongly associated with HIV-SN (AOR 2.5, 2.8 and 2.9 respectively).

Conclusions: HIV-SN is common among patients with advanced HIV infection in Cameroon. This simple diagnostic tool (BPNS) should therefore be routinely used to detect those with HIV-SN or at risk so as to minimise the negative impact it has on their quality of life.
\end{abstract}

Keywords: HIV-associated sensory neuropathy, HIV, CD4 count, Peripheral neuropathy

\section{Background}

Peripheral neuropathy (PN) which is the most common neurological complication in HIV infection [1] is widely under-recognised and under treated in resource constrained settings [2]. Clinically, there are at least six patterns of HIV associated peripheral neuropathy; distal sensory polyneuropathy (DSP), inflammatory demyelinating polyneuropathy

\footnotetext{
* Correspondence: hnluma@yahoo.com

${ }^{1}$ Department of internal Medicine, Douala General Hospital, Douala, Cameroon

${ }^{3}$ Faculty of Medicine and Biomedical Sciences, University of Yaoundé 1,

Yaoundé, Cameroon

Full list of author information is available at the end of the article
}

(IDP), progressive polyradiculopathy (PP), rapidly progressive flaccid paraparesis, mononeuritis multiplex (MM), and autonomic neuropathy [3]. Distal sensory peripheral neuropathy which is the most common of all HIV-associated sensory neuropathy (HIV-SN) [4] exists as two major types: primary HIV-associated distal sensory polyneuropathy (HIV-DSP), and antiretroviral toxic neuropathy (ATN), ATN being the most frequent antiretroviral therapy related toxicity in sub-Saharan Africa [5-8]. HIV-DSP and ATN together involve approximately $30-67 \%$ of patients with advanced HIV disease [1]. Despite significant improvement in the overall health of HIV-infected patients in the highly

\section{Biomed Central}


active antiretroviral therapy (HAART) era, HIV-SN still remains an important cause of morbidity among these patients because it considerably affects their quality of life $[9,10]$. The presence of HIV-SN-related neuropathic pain is a factor associated with greater unemployment, higher rates of depression and greater dependency in daily life activities albeit successful HAART [9]. More so, symptomatic relief therapy is usually not satisfactory [11]. In sub-Saharan Africa, many studies have not used a validated diagnostic tool to diagnose HIV-SN. In Cameroon, where the prevalence of HIV among adults has been estimated to be 5.3\% [12] the burden of HIV-SN has not yet been estimated. Furthermore, HAART is still composed of first line drugs some of which have been demonstrated in other studies to increase the risk of PN [13,14]. We therefore, decided to determine the prevalence of HIV-SN and its associated factors in HIV infected patients at the Douala General Hospital using an easy but validated screening tool, the Brief Peripheral Neuropathy Screening (BPNS) tool [15].

\section{Results}

\section{Patient characteristics}

A total of 295 adult HIV-1 infected patients were included in the study, 69.8\% (206/295) of who were females (Table 1). The mean age, weight and height were $42.3 \pm 10.4$ years, $70.6 \pm 12.8 \mathrm{~kg}$, and $1.6 \pm 0.1 \mathrm{~m}$ respectively. The median duration of HIV infection by $31^{\text {st }}$ October 2011 was 71 months (IQR 34.4 - 101.5). The median CD4 cell count was 200cells/ $\mu \mathrm{L}$ (IQR $102-300$ ). Of the 295 patients, 96.9\% (286) were on HAART, $45.4 \%(134 / 295)$ of who were on a regimen containing AZT-3TC-NVP (Table 1).

\section{HIV-associated sensory neuropathy (HIV-SN)}

Using the BPNS tool, the prevalence of neuropathic symptoms was $28.5 \%(84 / 295)$ the most common of which was sensation of pins and needles on legs/feet (Table 2). The median grade of symptoms on a scale of 0 to 10 was 4 (IQR 3 - 6). Among patients with symptoms, 36.9\% (31/ 84) had both abnormal reflexes and abnormal perceptions of tuning fork vibration, 19\% (16/84) had only abnormal reflexes, $17.9 \%(15 / 84)$ had only abnormal perception of vibrations and 26.2\% (22/84) had neither abnormal perception of vibration nor abnormal reflexes. Among those without symptoms, $27.5 \%(58 / 211)$ had at least an abnormal perception of vibrations or abnormal reflexes.

The prevalence of symptomatic HIV-SN was 21\% (62/ 295 ) of the study population. Among patients with symptomatic HIV-SN, the median duration of HIV infection was 79.8 months (IQR 46 - 107.5) with a median CD4 count of 153 cells/ $\mu \mathrm{L}$ (IQR $80-280$ ). Patient recall and clinical chart reviews, showed that $83.9 \%(52 / 62)$ had symptoms prior to HAART initiation with a median duration of symptoms of 24.3 months (IQR 10.8 - 44.7), the remaining $16.1 \%(10 / 62)$ developed symptoms after
Table 1 General characteristic of 295 patients with HIV 1 infection screened for HIV-SN

\begin{tabular}{ll}
\hline Characteristic & $\mathbf{n}(\%)$ \\
\hline Age group & \\
$<30$ & $34(11.5)$ \\
$30-39$ & $96(32.5)$ \\
$40-49$ & $94(31.9)$ \\
$50-59$ & $59(20.0)$ \\
$>60$ & $12(4.1)$ \\
\hline Sex & \\
Male & $89(30.2)$ \\
Female & $206(69.8)$ \\
\hline
\end{tabular}

CD4 count

$<200 \quad 143(48.5)$

$>200 \quad 152(51.5)$

CDC staging categories

A

A2 $36(12.2)$

A3 31 (10.5)

B1 $4(1.2)$

B2 $73(24.8)$

B3 $67(22.7)$

C1 $6(2.0)$

$\begin{array}{ll}\mathrm{C} 2 & 27(9.2)\end{array}$

C3 43 (14.6)

HAART regimen

AZT-3TC-EFV 46 (15.6)

AZT-3TC-NVP $134(45.4)$

TDF-3TC-EFV $22(7.5)$

TDF-FTC-EFV $4(1.4)$

D4T-3TC-NVP 46 (15.6)

Others 34 (11.5)

Not treated 9 (3.1)

History of anti-tuberculosis treatment

Yes 31 (10.5)

\begin{tabular}{ll} 
No & 264 (89.5) \\
\hline
\end{tabular}

History of alcohol intake

Yes $33(11.2)$

No 262 (88.8)

AZT: Zidovudine, 3TC: Lamivudine, EFV: Effavirenz, NVP: Nevirapine, D4T: Stavudine, TDF: Tenofovir.

HAART initiation with a median onset of symptoms of 17.7 months (IQR $2-41$ ).

\section{Association between HIV-SN and patient characteristics}

HIV-SN was strongly associated with advanced age, low CD4 count, history of alcohol consumption, history of anti-tuberculosis treatment but was modestly associated 
Table 2 Prevalence of signs and symptoms consistent with HIV-SN

\begin{tabular}{ll}
\hline Symptoms (at the legs or feet) & $\mathbf{n}(\%)$ \\
\hline Pain & $21(7.1)$ \\
Pins and needles & $39(13.2)$ \\
Numbness & $24(8.1)$ \\
Asymptomatic & $233(78.9)$ \\
\hline Ankle jerk reflexes compared to knee jerk & $41(13.9)$ \\
Grade 0: Absence of reflex & $48(16.3)$ \\
Grade 1: Hypoactive & $203(68.8)$ \\
Grade 2: Normal & $2(0.7)$ \\
Grade 3: Hyperactive & $1(0.3)$ \\
Grade 4: Clonus & \\
\hline Perception of tuning fork vibration on great toes & $213(72.2)$ \\
Grade 0: maximum perception for $>10$ s & $42(14.2)$ \\
Grade 1: perception for $6-10$ seconds & $25(8.5)$ \\
Grade 2: perception for <5 seconds & $15(5.1)$ \\
Grade 3: No perception &
\end{tabular}

with sex and height (Table 3). There was no association between HIV-SN and a history of D4T containing HAART regimens (Table 3).

After adjusting for age, sex and height, low CD4 count, history of anti-tuberculosis treatment and alcohol intake remained significantly associated with HIV-SN (Table 4).

\section{Discussion}

The definition of HIV-SN as per the BPNS tool assesses both subjective and objective findings consistent with PN. However, the requirements that a patient must have both neuropathic symptoms and clinical signs to be diagnosed as HIV-SN may exclude some patients with mild disease [16] indicating a substantial underestimation of PN [17]. This tool which is easy to use, practical and adds less than five minutes to the clinical examination of HIV patients during follow up in busy outpatient clinics [16] has been used in its standardised form in few studies. Using this tool, the prevalence of HIV-SN in our study population was $21 \%$ though, the prevalence of HIV-SN varies greatly among studies between 19 and 42\% [16,18-22]. According to Skopelitis and collaborators who used a model which involved both clinical criteria (symptoms and signs of neuropathy) and electrophysiology studies, two thirds of HIV-SN are subclinical [23]. This therefore means that some patients though asymptomatic might have abnormal signs relevant to HIV-SN but which do not meet up with HIV-SN diagnostic criteria as per the BPNS tool. This was the case in $19.7 \%$ (58/295) of our study population. Some authors $[19,20]$ considered this group as asymptomatic PN who perhaps represent those with
Table 3 Univariate analysis of possible associated factors for HIV-SN

\begin{tabular}{|c|c|c|c|c|}
\hline Variable & HIV-SN & No HIV-SN & OR $(95 \% \mathrm{Cl})$ & $P$ value \\
\hline \multicolumn{5}{|l|}{$\overline{\text { Age }}$} \\
\hline$<40$ & $16(25.8)$ & $114(48.9)$ & 2.8 & 0.001 \\
\hline$>40$ & $46(74.2)$ & $119(51.1)$ & $(1.5-5.2)$ & \\
\hline \multicolumn{5}{|l|}{$\overline{\text { Sex }}$} \\
\hline Male & $25(40.3)$ & $64(27.5)$ & 1.8 & 0.05 \\
\hline Female & $37(59.7)$ & $169(72.5)$ & $(1-3.2)$ & \\
\hline \multicolumn{5}{|c|}{ CD4 cell count } \\
\hline$<200$ & $40(64.5)$ & $103(44.2)$ & 2.3 & 0.004 \\
\hline$>200$ & $22(35.5)$ & $130(55.8)$ & $(1.3-4.1)$ & \\
\hline \multicolumn{5}{|l|}{ Height } \\
\hline$<1.7 \mathrm{~m}$ & $38(61.3)$ & $171(73.4)$ & 1.7 & 0.06 \\
\hline$>1.7 \mathrm{~m}$ & $24(38.7)$ & $62(26.6)$ & $(1-3.1)$ & \\
\hline \multicolumn{5}{|c|}{ History of alcohol intake } \\
\hline Yes & $13(21)$ & $20(8.6)$ & 2.8 & 0.006 \\
\hline No & $49(79)$ & $213(91.4)$ & $(1.3-6.1)$ & \\
\hline \multicolumn{5}{|c|}{ History of anti TB treatment } \\
\hline Yes & $11(17.7)$ & $20(8.6)$ & 2.3 & 0.04 \\
\hline No & $51(82.3)$ & $213(91.4)$ & $(1.0-5.1)$ & \\
\hline \multicolumn{5}{|c|}{ AZT in HAART regimen } \\
\hline Yes & $26(41.9)$ & $89(38.2)$ & 0.9 & 0.6 \\
\hline No & $36(58.1)$ & $144(61.8)$ & $(0.5-1.5)$ & \\
\hline \multicolumn{5}{|c|}{ D4T in HAART regimen } \\
\hline Yes & $12(19.4)$ & $34(14.6)$ & 1.4 & 0.4 \\
\hline No & $50(80.7)$ & $199(85.4)$ & $(0.7-2.9)$ & \\
\hline Total & $62(21)$ & $233(79)$ & / & / \\
\hline
\end{tabular}

early HIV-SN who are more likely to become symptomatic when challenged with HAART or other risks for PN [20]. Considering together those with HIV-SN (according to BPNS criteria) as symptomatic PN patients and those with clinical abnormalities relevant with $\mathrm{PN}$ but no symptoms as asymptomatic PN patients, increases the prevalence of $\mathrm{PN}$ in our study to $40.7 \%$. In a resource limited setting like ours, this approach increases the likelihood of detection of patients with or at risk of PN. Consequently, caregiver choice of drugs of the HAART regimen would take into consideration each patient's risk of PN and patient education on the avoidable risk factors would be accentuated. Either way HIV$\mathrm{SN}$ is common among patients with HIV in Cameroon.

We found that HIV-SN is more common among older patients, similar with findings in other studies $[4,16,19$ $21,24]$. This is because peripheral nerves by their length and size are known to have increased vulnerability with aging due to continual metabolic stress and exposure to toxic substances of which alcohol is among the most common as could be seen in our study where after 
Table 4 Multivariate analysis of associated factors for HIV-SN adjusted for age, sex and height

\begin{tabular}{llll}
\hline & Adjusted Odd ratio $($ AOR $)$ & 95\% Cl & P value \\
\hline Low CD4 $\left(<200 / \mathrm{mm}^{3}\right)$ & 2.5 & $1.3-4.6$ & 0.003 \\
History of alcohol intake & 2.8 & $1.2-6.6$ & 0.01 \\
History of anti TB treatment & 2.9 & $1.3-6.8$ & 0.01 \\
\hline
\end{tabular}

adjusting for age, sex and height alcohol intake still remained strongly associated with HIV-SN consistent with findings in similar settings like ours [22]. Morgello and collaborators in America also found that HIV-SN correlated with alcohol intake. Furthermore, increased life expectancy in the HAART era predisposes to longer exposure to the virus and/or to dideoxynucleoside analogues [23]. Even though, there is considerable evidence on the risk of PN in the HAART era with use of neurotoxic agents $[4,17,25]$, our study failed to show any such association especially with D4T. Another study [19] found that pre-existing PN before starting HAART reduced the risk that a patient would be placed on a D4T based regimen [19] thereby iterating the importance of pre-HAART screening for PN. However, the absence of association between D4T use and PN in our study could have been because only $15.6 \%$ of our patients were on a D4T containing regimen compared to as much as $98 \%$ in other studies in similar limited resource settings $[19,21]$.

A history of anti-tuberculosis treatment was found to be strongly associated to HIV-SN in our study. Isoniazid which is a major component of antiTB treatment during the whole treatment period has been shown to be a risk factor of PN [1].

Finally, our finding of low CD4 count, a reflection of advanced HIV disease, to be strongly associated with HIV-SN, was in line with findings in many other studies $[4,16,17,19,21,24]$. Moreover, given that $83.9 \%$ of our symptomatic patients had symptoms before HAART initiation could be evidence that HIV-SN primarily might have been be due to the virus (HIV-DSP) [20].

Our study had some limitations. Firstly being a hospital based study in a tertiary institution where care is expensive; there is possibility of selection bias not permitting us to capture the real picture of HIV-SN among HIV patients in Cameroon. Secondly, a longitudinal design, unlike our cross-sectional design would enable us determine the incidence of ATN most especially as HAART coverage is being scaled up.

\section{Conclusions}

HIV-SN is common among HIV 1-infected patients at the Douala General Hospital. Simple neurological evaluation tools to detect early HIV-SN or those at risk among all HIV patients should be routinely used to diagnose this ailment which negatively impacts their quality of life. HIV-SN increases in frequency with severity of immune depression thereby necessitating early diagnosis and treatment of HIV to avoid severe immune depression. Finally, clinicians' choice of drugs of the HAART regimen and other drugs for HIV comorbidities should consider the role these drugs play as a factor associated with HIV-SN most especially as we found that a proportion of our patients developed symptoms relevant with HIV-SN only after HAART initiation and use of antituberculosis drugs.

\section{Methods}

\section{Study setting and patients}

After prior local institutional ethical clearance, we carried out a cross sectional study at the adult HIV outpatient clinic of the Douala General Hospital, a tertiary hospital with a capacity of 320beds, situated in Douala, the economic capital of Cameroon between $1^{\text {st }}$ July and $31^{\text {st }}$ October 2011. Our study population comprised of consenting adults ( $>18$ years) diagnosed with HIV and followed up in this hospital. HIV diagnosis in this institution is made according to Cameroon National AIDS Control Programme guidelines [26] by antibody detection on two successive samples using a third generation ELISA test BIOREX $^{\circledR}$ (Biorex Diagnostics Limited, Antrim, United Kingdom). If both are positive, a third sample is collected and tested using Genie ${ }^{\circledR}$ III HIV-1/HIV-2 Assay (Bio-Rad Diagnostics, Marnes la Coquette, France) to specify either HIV 1 or HIV 2. A patient is declared positive for HIV if these three tests are positive. In case of any discordance, testing is done with Western blot (New LAV blot, Diagnostics, Pasteur, Marnes la Coquette, France).

\section{Study procedure}

Consecutively, each consenting patient underwent a brief peripheral neuropathy screening (BPNS) using the BPNS tool validated by the AIDS Clinical Trial Group (ACTG) [15]. This consists of brief questions regarding symptoms of HIV-SN: pain, aching or burning sensation in the feet/ legs, pins and needles sensation in the feet/legs and numbness in the feet/legs. Screening was performed by a final year medical student trained for the purpose. Each symptom when present was subjectively graded bilaterally from 0 (absent) to 10 (severe). Lower extremity examination was then done to evaluate patient perception of vibrations for over 10seconds using a $128 \mathrm{~Hz}$ tuning fork on the big toe. Ankle reflexes were also tested using a reflex hammer. 
Both findings were graded as follows: vibrations (grade 0: maximum perception for $>10$ s, grade $1: 6-10$ s, grade 2 : $<5$ s, grade 3: no perception) and ankle reflexes (grade 0 : absent, grade 1: hypoactive, grade 2: normal, grade 3 : hyperactive, grade 4 : clonus). To ensure proper examination, random patients were chosen for examination by the neurologist whose findings were compared to those of the student for concordance and all were concordant. Socio-demographic and anthropometric information relevant to the study were also obtained from each patient and completed by clinical case files examination.

\section{Statistical analysis}

Data entry was in Epi Data 3.1 and analysis in STATA 11.2 (Stata Corporation, College Station, Texas). HIV-SN was defined by the presence of symptoms and at least an abnormal perception of vibrations or abnormal ankle reflexes or both and expressed as a percentage of the study population. It was then categorised as either present or absent and compared to other variables using chi square test and Fisher's exact test where necessary. Mantel Haenszel method was used to measure strength of association between HIV-SN and covariates and a final model was made using logistic regression. Crude and adjusted Odd Ratios (OR) together with its 95\% Confidence Intervals $(95 \% \mathrm{CI})$ was reported. Evidence of association was considered for a two-sided $p$ value of less than 0.05 .

\section{Competing interests}

All authors declare no conflict of interest.

\section{Authors' contributions}

$H N L, B C N T, M S D, E T$ and VPD designed the study. VNKS, BCNT, and YNM collected and entered data. HNL, BCNT and ET did data analysis and manuscript write-up. VPD and MSD did manuscript proofreading and editing. All authors agreed with the final manuscript to be submitted. All authors read and approved the final manuscript.

\section{Acknowledgements}

The authors wish to sincerely thank all the member of the HIV/AIDS therapeutic committee of the Douala General Hospital, most especially Dr Henry Achu Joko. We are also grateful to members of the Doula Research Network (DRN) for being the critics of the study protocol.

\section{Author details \\ 'Department of internal Medicine, Douala General Hospital, Douala, Cameroon. ' ${ }^{2}$ epartment of Neurosurgery, Yaoundé Central Hospital, Yaoundé, Cameroon. ${ }^{3}$ Faculty of Medicine and Biomedical Sciences, University of Yaoundé 1, Yaoundé, Cameroon. ${ }^{4}$ Université des Montagnes, Bagangte, Cameroon.}

Received: 8 October 2012 Accepted: 23 November 2012 Published: 26 November 2012

\section{References}

1. Kamerman PR, Wadley AL, Cherry CL: HIV-associated sensory neuropathy: risk factors and genetics. Current pain and headache reports 2012, 16(3):226-236.

2. Beadles WI, Jahn A, Weigel R, Clutterbuck D: Peripheral neuropathy in HIV-positive patients at an antiretroviral clinic in lilongwe, malawi. Tropical doctor 2009, 39(2):78-80.
3. Wulff EA, Wang AK, Simpson DM: HIV-associated peripheral neuropathy: epidemiology, pathophysiology and treatment. Drugs 2000, 59(6):1251-1260

4. Morgello S, Estanislao L, Simpson D, Geraci A, DiRocco A, Gerits P, Ryan E, Yakoushina T, Khan S, Mahboob R, Naseer M, Dorfman D, Sharp V: HIVassociated distal sensory polyneuropathy in the era of highly active antiretroviral therapy: the manhattan HIV brain bank. Arch Neurol 2004, 61(4):546-551.

5. WHO/Forum for collaborative HIV Research Joint Meeting: ARV drugs adverse events, case definition, grading, laboratory diagnosis and treatment monitoring. Geneva, Switzerland; 2008. http://www.hivforum.org/index.php? option=com_content\&task=view\&id=59\&ltemid=102. Accessed on 16/10/2011.

6. Modayil RR, Harugeri A, Parthasarathi G, Ramesh M, Prasad R, Naik V, Giriyapura V: Adverse drug reactions to antiretroviral therapy (ART): an experience of spontaneous reporting and intensive monitoring from ART centre in india. Pharmacoepidemiology and drug safety 2010, 19(3):247-255.

7. Hawkins C, Achenbach C, Fryda W, Ngare D, Murphy R: Antiretroviral durability and tolerability in HIV-infected adults living in urban kenya. J Acquir Immune Defic Syndr 2007, 45(3):304-310.

8. Forna F, Liechty CA, Solberg P, Asiimwe F, Were W, Mermin J, Behumbiize P, Tong T, Brooks JT, Weidle PJ: Clinical toxicity of highly active antiretroviral therapy in a home-based AIDS care program in rural uganda. J Acquir Immune Defic Syndr 2007, 44(4):456-462.

9. Ellis RJ, Rosario D, Clifford DB, MCArthur JC, Simpson D, Alexander T, Gelman BB, Vaida F, Collier A, Marra CM, Ances B, Atkinson JH, Dworkin RH, Morgello $S$, Grant I: Continued high prevalence and adverse clinical impact of human immunodeficiency virus-associated sensory neuropathy in the era of combination antiretroviral therapy: the CHARTER study. Arch Neurol 2010, 67(5):552-558.

10. Robinson-Papp J, Morgello S, Vaida F, Fitzsimons C, Simpson DM, Elliott KJ, Al-Lozi M, Gelman BB, Clifford D, Marra CM, McCutchan JA, Atkinson JH, Dworkin RH, Grant I, Ellis R: Association of self-reported painful symptoms with clinical and neurophysiologic signs in HIV-associated sensory neuropathy. Pain 2010, 151(3):732-736.

11. Phillips TJ, Cherry CL, Cox S, Marshall SJ, Rice AS: Pharmacological treatment of painful HIV-associated sensory neuropathy: a systematic review and meta-analysis of randomised controlled trials. PLoS One 2010, 5(12):e14433.

12. UNAIDS: Report on the global HIV/AIDS epidemic. Geneva, Switzerland: Joint United Nations Programme on HIV/AIDS; 2008.

13. Simpson DM, Tagliati M: Nucleoside analogue-associated peripheral neuropathy in human immunodeficiency virus infection. Journal of acquired immune deficiency syndromes and human retrovirology: official publication of the International Retrovirology Association 1995, 9(2):153-161.

14. Moore RD, Wong WM, Keruly JC, McArthur JC: Incidence of neuropathy in HIV-infected patients on monotherapy versus those on combination therapy with didanosine, stavudine and hydroxyurea. AIDS 2000, 14(3):273-278.

15. Cherry CL, Wesselingh SL, Lal L, McArthur JC: Evaluation of a clinical screening tool for HIV-associated sensory neuropathies. Neurology 2005, 65(11):1778-1781.

16. Cherry CL, Affandi JS, Imran D, Yunihastuti E, Smyth K, Vanar S, Kamarulzaman A, Price P: Age and height predict neuropathy risk in patients with HIV prescribed stavudine. Neurology 2009, 73(4):315-320.

17. Evans D, Takuva S, Rassool M, Firnhaber C, Maskew M: Prevalence of peripheral neuropathy in antiretroviral therapy naive HIV-positive patients and the impact on treatment outcomes-a retrospective study from a large urban cohort in Johannesburg, south Africa. Journal of neurovirology 2012, 18(3):162-171.

18. Cettomai D, Kwasa J, Kendi C, Birbeck GL, Price RW, Bukusi EA, Cohen CR, Meyer AC: Utility of quantitative sensory testing and screening tools in identifying HIV-associated peripheral neuropathy in western kenya: pilot testing. PLoS One 2010, 5(12):e14256.

19. Mehta SA, Ahmed A, Kariuki BW, Said S, Omasete F, Mendillo M, Laverty M, Holzman R, Valentine F, Sivapalasingam S: Implementation of a validated peripheral neuropathy screening tool in patients receiving antiretroviral therapy in mombasa, kenya. AmJTrop Med Hyg 2010, 83(3):565-570.

20. Evans SR, Ellis RJ, Chen H, Yeh TM, Lee AJ, Schifitto G, Wu K, Bosch RJ, MCArthur JC, Simpson DM, Clifford DB: Peripheral neuropathy in HIV: prevalence and risk factors. AIDS 2011, 25(7):919-928. 
21. Smyth K, Affandi JS, McArthur JC, Bowtell-Harris C, Mijch AM, Watson K, Costello K, Woolley IJ, Price P, Wesselingh SL, Cherry CL: Prevalence of and risk factors for HIV-associated neuropathy in melbourne, australia 1993-2006. HIV medicine 2007, 8(6):367-373.

22. Maritz J, Benatar M, Dave JA, Harrison TB, Badri M, Levitt NS, Heckmann JM: HIV neuropathy in south africans: frequency, characteristics, and risk factors. Muscle Nerve 2010, 41(5):599-606.

23. Skopelitis EE, Kokotis PI, Kontos AN, Panayiotakopoulos GD, Konstantinou K Kordossis T, Karandreas N: Distal sensory polyneuropathy in HIV-positive patients in the HAART era: an entity underestimated by clinical examination. Int J STD AIDS 2006, 17(7):467-472.

24. Watters MR, Poff PW, Shiramizu BT, Holck PS, Fast KM, Shikuma CM, Valcour VG: Symptomatic distal sensory polyneuropathy in HIV after age 50 . Neurology 2004, 62(8):1378-1383.

25. Menezes CN, Maskew M, Sanne I, Crowther NJ, Raal FJ: A longitudinal study of stavudine-associated toxicities in a large cohort of south african HIV infected subjects. BMC Infect Dis 2011, 11:244

26. Plan strategique national de lutte contre le vih, le sida et les ist; 2011 Available from: http://www.circb.com/doc/PSN\%202011-2015.pdf.

doi:10.1186/1742-6405-9-35

Cite this article as: Luma et al.: HIV-associated sensory neuropathy in HIV-1 infected patients at the Douala General Hospital in Cameroon: a cross-sectional study. AIDS Research and Therapy 2012 9:35.

\section{Submit your next manuscript to BioMed Central and take full advantage of:}

- Convenient online submission

- Thorough peer review

- No space constraints or color figure charges

- Immediate publication on acceptance

- Inclusion in PubMed, CAS, Scopus and Google Scholar

- Research which is freely available for redistribution 